\title{
A new technique of Laplace Padé reduced differential transform method for $(1+3)$ dimensional wave equations
}

\author{
Omer Acan ${ }^{1,2}$ and Yildiray Keskin ${ }^{2}$ \\ ${ }^{1}$ Department of Mathematics, Faculty of Arts and Science, Siirt University, Siirt, Turkey \\ ${ }^{2}$ Department of Mathematics, Science Faculty, Selcuk University, Konya, Turkey
}

Received: 27 March 2016, Accepted: 5 June 2016

Published online: 15 February 2017.

\begin{abstract}
The aim of this paper is to give a good strategy for solving some linear and non-linear partial differential equations in mechanics, physics, engineering and various other technical fields by Modified Reduced Differential Transform Method. In this article we use the method named with Laplace-Padé Reduced Differential Transform Method. This method is obtained by combining LaplacePadé resummation method, which is a useful technique to find exact solutions, and the Reduced Differential Transform Method. We apply the method to the wave equations and give some examples to see its effectiveness and usefulness. The results and the findings showed that this method leads us to exact solutions with a few iterations or the approximate solutions with small errors.
\end{abstract}

Keywords: Laplace-Padé Reduced Differential Transform Method (LPRDTM), Modified Reduced Differential Transform Method (MRDTM), Reduced Differential Transform Method (RDTM), Partial differential equations (PDEs), wave equation.

\section{Introduction}

As widely known, the importance of research linear and non-linear PDEs have a large number of essential application studies in different branches of engineering and science such as fluid physics, plasma physics, non-linear fiberoptics, fluidmechanics, thermodynamic mechanic, thermodynamic, heat transfer, oceanography and atmospheric science[1-3]. Many researchers have paid attention to the solutions of linear and non-linear PDEs by various methods, such as, the differential transform method (DTM)[4-7], RDTM [8-15], the variational iteration method (VIM)[16,17] the homotopy analysis method (HAM)[18-20] and the Adomian decomposition method[21] among others.

Consider the following general form of $(1+3)$ dimensional wave Equation,

$$
u_{t t}+a(x, y, z, t) u_{x x}+b(x, y, z, t) u_{y y}+c(x, y, z, t) u_{z z}=e(x, y, z, t)
$$

subject to initial condition

$$
\begin{aligned}
& u(x, y, z, 0)=f(x, y, z) \\
& u_{t}(x, y, z, 0)=g(x, y, z) .
\end{aligned}
$$

We apply LPRDTM (combining Laplace-Padé resummation method and RDTM ) to solve wave equation of the form (1).

This study is organized as follows. In Section 2, we briefly describe LPRDTM. Two numerical examples are introduced in Section 3 for demonstrating the complete study. Conclusion is given in the last section. 


\section{Laplace-Padé reduced differential transform method (LPRDTM)}

\subsection{Reduced Differential Transform Method (RDTM)}

We will briefly introduce RDTM [8] for wave equation in this section. We consider the wave equation in the operator form

$$
L_{t t}(u(x, y, z, t))+L(u(x, y, z, t))=e(x, y, z, t)
$$

with initial conditions

$$
\begin{aligned}
u(x, y, z, 0) & =f(x, y, z) \\
u_{t}(x, y, z, 0) & =g(x, y, z)
\end{aligned}
$$

where $L_{t t}(u(x, y, z, t))=u_{t t}$ and $L(u(x, y, z, t))=a(x, y, z, t) u_{x x}+b(x, y, z, t) u_{y y}+c(x, y, z, t) u_{z z}$ are linear operators.

Definition 1. If $u(x, y, z, t)$ is differentiated continuously with respect to time tand analyticfunction and spaces $x, y$ and $z$ in the domain of interest, then the spectrum function

$$
U_{k}(x, y, z)=\frac{1}{k !}\left[\frac{\partial^{k}}{\partial t^{k}} u(x, y, z, t)\right]_{t=0}
$$

is the reduced transformed function of $u(x, y, z, t)$. In this study, the lowercase $u(x, y, z, t)$ represent the original function while the uppercase $U_{k}(x, y, z)$ stand for the transformed function. The differential inverse transform of $U_{k}(x, y, z)$ is defined as.

$$
u(x, y, z, t)=\sum_{k=0}^{\infty} U_{k}(x, y, z) t^{k}
$$

Combining equation (5) and (6). it can be obtained that

$$
u(x, y, z, t)=\sum_{k=0}^{\infty} \frac{1}{k !}\left[\frac{\partial^{k}}{\partial t^{k}} u(x, y, z, t)\right]_{t=0} t^{k}
$$

From the above definition, one can find that the concept of the reduced $(1+3)$ dimensional differential transform is derived from the power series expansion. The following theorem of the fundamental operators of RDTM is given below (for details see[8-10].

Theorem 1. Assume that the reduced differential transform functions of $u(x, y, z, t), v(x, y, z, t)$ and $w(x, y, z, t)$ are $U_{k}(x, y, z), V_{k}(x, y, z)$ and $W_{k}(x, y, z)$ respectively. Then,

(i) If $w(x, y, z, t)=u(x, y, z, t) \pm \alpha v(x, y, z, t)$, then $W_{k}(x, y, z)=U_{k}(x, y, z) \pm \alpha V_{k}(x, y, z)(\alpha$ is a constant),

(ii) If $w(x, y, z, t)=u(x, y, z, t) v(x, y, z, t)$, then $W_{k}(x, y, z)=\sum_{r=0}^{k} V_{r}(x, y, z) U_{k-r}(x, y, z)=\sum_{r=0}^{k} U_{r}(x, y, z) V_{k-r}(x, y, z)$,

(iii) If $w(x, y, z, t)=\frac{\partial^{r}}{\partial t^{r}} u(x, y, z, t)$, then $W_{k}(x, y, z)=(k+1) \ldots(k+r) U_{k+r}(x, y, z)=\frac{(k+r) !}{k !} U_{k+r}(x, y, z)$,

(iv) If $w(x, y, z, t)=x^{m} y^{n} z^{q} t^{p}$, then $W_{k}(x, y, z)=\left\{\begin{array}{l}x^{m} y^{n} z^{q}, k=p \\ 0, k \neq p\end{array}\right.$,

(v) If $w(x, y, z, t)=\frac{\partial}{\partial x^{m} \partial y^{n} \partial z^{q}} u(x, y, z, t)$, then $W_{k}(x, y, z)=\frac{\partial}{\partial x^{m} \partial y^{n} \partial z^{q}} U_{k}(x, y, z)$.

Assume that the reduced differential transform functions of $a(x, y, z, t), b(x, y, z, t), c(x, y, z, t)$ and $e(x, y, z, t)$ $\operatorname{are}_{k}(x, y, z), B_{k}(x, y, z), C_{k}(x, y, z) \operatorname{andE}_{k}(x, y, z)$ respectively. Then, According to Theorem 1 and the RDTM, from (3) we can construct the following iteration formula.

$$
\begin{array}{r}
(k+1)(k+2) U_{k+2}(x, y, z)=E_{k}(x, y, z)-\sum_{r=0}^{k} A_{r}(x, y, z) \frac{\partial}{\partial x^{2}} U_{k-r}(x, y, z) \\
-\sum_{r=0}^{k} B_{r}(x, y, z) \frac{\partial}{\partial y^{2}} U_{k-r}(x, y, z)-\sum_{r=0}^{k} C_{r}(x, y, z) \frac{\partial}{\partial z^{2}} U_{k-r}(x, y, z)
\end{array}
$$


From initial condition (3), we write

$$
\begin{aligned}
& U_{0}(x, y, z)=f(x, y, z) \\
& U_{1}(x, y, z)=g(x, y, z)
\end{aligned}
$$

Substituting (9) into (8) and by a straight forward iterative calculations, we get the following $U_{k}(x, y, z)$ values. Then the inverse transformation of the set of values $\left\{U_{k}(x, y, z)\right\}_{k=0}^{n}$ gives approximation solution as,

$$
\tilde{u}_{n}(x, y, z, t)=\sum_{k=0}^{n} U_{k}(x, y, z) t^{k}
$$

where $n$ is order of approximation solution. Therefore, the exact solution of problem is given by

$$
u(x, y, z, t)=\lim _{n \rightarrow \infty} \tilde{u}_{n}(x, y, z, t)
$$

\subsection{Padé Approximant}

Let $u(x, y, z, t)$ be an analytical function with Maclaurin's expansion

$$
u(x, y, z, t)=\sum_{k=0}^{n} u_{n} t^{k}
$$

Then the Padé approximant to $u(x, y, z, t)$ of order $[K, L]$ which we denote by $[K / L]_{u}(x, y, z, t)$ is defined by [22,23],

$$
\left[\frac{K}{L}\right]_{u}(x, y, z, t)=\frac{p_{0}+p_{1} t+\cdots+p_{L} t^{K}}{q_{0}+q_{1} t+\cdots+q_{M} t^{L}},
$$

where $q_{0}=1$, and the numerator and denominator polynomials have no common factors. The numerator and denominator polynomials are constructed as follows

$$
u(x, y, z, t)-\left[\frac{K}{L}\right]_{u}(x, y, z, t)=O\left(t^{K+L+1}\right)
$$

From (14), it is obtained

$$
u(x, y, z, t) \sum_{n=0}^{L} q_{n} t^{n}-\sum_{n=0}^{K} p_{n} t^{n}=O\left(t^{K+L+1}\right) .
$$

From (15), the following algebraic linear systems are obtained as

$$
\begin{gathered}
u_{K} q_{1}+\cdots+u_{K-L+1} q_{M}=-u_{K+1}, \\
u_{K+1} q_{1}+\cdots+u_{K-L+2} q_{M}=-u_{K+2}, \\
\vdots \\
u_{K+L-1} q_{1}+\cdots+u_{K} q_{L}=-u_{K+L}, \\
p_{0}=u_{0} \\
p_{1}=u_{1}+u_{0} q_{1} \\
\vdots \\
p_{K}=u_{K}+u_{K-1} q_{1}+\cdots+u_{0} q_{K} .
\end{gathered}
$$


From (16), we calculate first all the coefficients $q_{n}, 1 \leq n \leq L$, Then, we determine the coefficients $p_{n}, 0 \leq n \leq K$, from (17).

\subsection{Laplace-Padé reduced differential transform method (LPRDTM)}

Some numerical methods give power series solutions. However, this kind of solutions have narrow domains of convergence. Hence, Laplace-Padé [23-26] resummation method is used to extend the domain of convergence of the solutions or to obtain exact solutions. We describe the LPRDTM which is combination of RDTM and Laplace-Padé resummation method as follows.

(i) Firstly, let's get series as in (2.8) by using RDTM up to a certain iteration for the initial value problem.

(ii) Secondly, we apply Laplace transformation to the obtained series (10).

(iii) Next, we write $1 /$ tinstead of $s$ in the obtained equation.

(iv) After that, we transform the series obtained from (iii) into a meromorphic function by creating its Padé approximant of order $[K / L]$ where $K$ and $L$ are randomly chosen, but they should be smaller than the order of the series. In this step, the Padé approximant enlarges the domain of the obtained series solution to get better convergence and accuracy.

(v) Then, we write $1 /$ sinstead of $t$ in the obtained equation.

(vi) Finally, we get the approximate or exact solution by using the inverse Laplace $s$ transformation.

\section{Numerical consideration}

We consider two examples applied to LPRDTM.

Example 1. Considering the following wave equation,

$$
u_{t t}=\frac{1}{6}\left(x^{2} u_{x x}+y^{2} u_{y y}+z^{2} u_{z z}\right)
$$

subject to initial conditions:

$$
\begin{aligned}
& u(x, y, z, 0)=x^{2} y^{2} z^{2}, \\
& u_{t}(x, y, z, 0)=-x^{2} y^{2} z^{2} .
\end{aligned}
$$

Now we apply the steps $(i)-(v i)$ to our example. If we apply RDTM and Theorem 2.1 in (3.1) it gives us

$$
U_{k+2}(x, y, z)=\frac{1}{6(k+1)(k+2)}\left(x^{2} \frac{\partial^{2}}{\partial x^{2}} U_{k}(x, y, z)+y^{2} \frac{\partial^{2}}{\partial y^{2}} U_{k}(x, y, z)+z^{2} \frac{\partial^{2}}{\partial z^{2}} U_{k}(x, y, z)\right)
$$

where $U_{k}(x, y, z)$ 's are the transformed functions. By the initial conditions (19) we write

$$
\begin{aligned}
& U_{0}(x, y, z)=x^{2} y^{2} z^{2} \\
& U_{1}(x, y, z)=-x^{2} y^{2} z^{2}
\end{aligned}
$$

By substituting (21) into (20) respectively, we obtain

$$
\begin{gathered}
U_{2}(x, y, z)=\frac{x^{2} y^{2} z^{2}}{2} \\
U_{3}(x, y, z)=-\frac{x^{2} y^{2} z^{2}}{6},
\end{gathered}
$$




$$
U_{4}(x, y, z)=\frac{x^{2} y^{2} z^{2}}{24}
$$

By (10)

$$
\tilde{u}_{4}(x, y, z, t)=\sum_{k=0}^{4} U_{k}(x, y, z) t^{k} .
$$

Substituting (21), (22), (23) and (24) in (25), we get

$$
\tilde{u}_{4}(x, y, z, t)=x^{2} y^{2} z^{2}-x^{2} y^{2} z^{2} t+\frac{x^{2} y^{2} z^{2}}{2} t^{2}-\frac{x^{2} y^{2} z^{2}}{6} t^{3}+\frac{x^{2} y^{2} z^{2}}{24} t^{4} .
$$

If we apply Laplace transform to $\tilde{u}_{4}(x, y, z, t)$, it gives

$$
L\left[\tilde{u}_{4}(x, y, z, t)\right]=\frac{x^{2} y^{2} z^{2}\left(s^{4}-s^{3}+s^{2}-s+1\right)}{s^{5}} .
$$

We write $1 / t$ instead of $\mathrm{s}$ in (27) then

$$
L\left[\tilde{u}_{4}(x, y, z, t)\right]=x^{2} y^{2} z^{2}\left(1-t+t^{2}-t^{3}+t^{4}\right) t .
$$

All $[K / L]$-Padé approximants of (28) with $L \geq 1, K \geq 1$ and $K+L \leq 4$ give

$$
\left[\frac{K}{L}\right]_{\tilde{u}_{4}}(x, y, z, t)=\frac{x^{2} y^{2} z^{2} t}{1+t} .
$$

Now by changing $1 / s$ into $t$ in (3.12), we obtain $[K / L]_{\tilde{u}_{4}}$ in terms of $s$ as follow,

$$
\left[\frac{L}{M}\right]_{\tilde{u}_{4}}(x, y, z, t)=\frac{x^{2} y^{2} z^{2}}{1+s} .
$$

Finally, when we apply the inverse Laplace transform to (3.13), it gives us an approximate solution. In fact, the approximate solution corresponds the exact solution.

$$
\tilde{u}_{4}(x, y, z, t)=u(x, y, z, t)=x^{2} y^{2} z^{2} e^{-t} .
$$

Example 2. Now we consider the following another wave equation.

$$
u_{t t}=a u_{x x}+b u_{y y}+c u_{z z}
$$

where $a, b$ and $c$ are constant. Subject to initial conditions.

$$
\begin{aligned}
u(x, y, z, 0) & =a_{1} x+a_{2} x^{2}+b_{1} y+b_{2} y^{2}+c_{1} z+c_{2} z^{2}, \\
u_{t}(x, y, z, 0) & =a^{\prime}{ }_{1} x+a^{\prime}{ }_{2} x^{2}+b^{\prime}{ }_{1} y+b^{\prime}{ }_{2} y^{2}+c^{\prime}{ }_{1} z+c^{\prime}{ }_{2} z^{2} .
\end{aligned}
$$

Now we apply the steps $(i)-(v i)$ to our example. If we apply RDTM and Theorem 1 in (32) it gives us

$$
U_{k+2}(x, y, z)=\frac{1}{(k+1)(k+2)}\left(a \frac{\partial^{2}}{\partial x^{2}} U_{k}(x, y, z)+b \frac{\partial^{2}}{\partial y^{2}} U_{k}(x, y, z)+c \frac{\partial^{2}}{\partial z^{2}} U_{k}(x, y, z)\right)
$$


where $U_{k}(x, y, z)$ 's are the transformed functions. By the initial conditions (33) we write

$$
\begin{aligned}
& U_{0}(x, y, z)=a_{1} x+a_{2} x^{2}+b_{1} y+b_{2} y^{2}+c_{1} z+c_{2} z^{2} \\
& U_{1}(x, y, z)=a^{\prime}{ }_{1} x+a_{2}{ }_{2} x^{2}+b^{\prime}{ }_{1} y+b^{\prime}{ }_{2} y^{2}+c^{\prime}{ }_{1} z+c^{\prime}{ }_{2} z^{2} .
\end{aligned}
$$

Now, substituting (35) into (34) respectively, we obtain

$$
\begin{gathered}
U_{2}(x, y, z)=a a_{2}+b b_{2}+c c_{2} \\
U_{3}(x, y, z)=\frac{1}{3}\left(a a_{2}^{\prime}+b b_{2}^{\prime}+c c^{\prime}{ }_{2}\right), \\
U_{4}(x, y, z)=0 .
\end{gathered}
$$

By (10)

$$
\tilde{u}_{4}(x, y, z, t)=\sum_{k=0}^{4} U_{k}(x, y, z) t^{k} .
$$

Substituting (35), (36), (37) and (38) in (39), we get

$$
\begin{aligned}
\tilde{u}_{4}(x, y, z, t) & =a_{1} x+a_{2} x^{2}+b_{1} y+b_{2} y^{2}+c_{1} z+c_{2} z^{2}+\left(a_{1}^{\prime} x+a_{2}^{\prime} x^{2}+b^{\prime}{ }_{1} y+b_{2}^{\prime} y^{2}+c^{\prime}{ }_{1} z+c^{\prime}{ }_{2} z^{2} .\right) t \\
& +\left(a a_{2}+b b_{2}+c c_{2}\right) t^{2}+\frac{1}{3}\left(a a_{2}^{\prime}+b b_{2}^{\prime}+c c_{2}^{\prime}\right) t^{3}
\end{aligned}
$$

If we apply Laplace transform to $\tilde{u}_{4}(x, y, z, t)$, it gives

$$
\begin{aligned}
L\left[\tilde{u}_{4}(x, y, z, t)\right] & =\frac{a_{1} x+a_{2} x^{2}+b_{1} y+b_{2} y^{2}+c_{1} z+c_{2} z^{2}}{s}+\frac{a_{1}^{\prime} x+a_{2}^{\prime} x^{2}+b^{\prime}{ }_{1} y+b_{2}^{\prime} y^{2}+c^{\prime}{ }_{1} z+c^{\prime}{ }_{2} z^{2}}{s^{2}} \\
& +\frac{2 a a_{2}+2 b b_{2}+2 c c_{2}}{s^{3}}+\frac{2 a a_{2}^{\prime}+2 b b_{2}^{\prime}+2 c c_{2}^{\prime}}{s^{4}} .
\end{aligned}
$$

We write $1 / t$ instead of $s$ in (27) then

$$
L\left[\tilde{u}_{4}(x, y, z, t)\right]=\left(\begin{array}{l}
a_{1} x+a_{2} x^{2}+b_{1} y+b_{2} y^{2}+c_{1} z+c_{2} z^{2}+\left(a_{1}^{\prime} x+a_{2}^{\prime} x^{2}+b^{\prime}{ }_{1} y+b^{\prime}{ }_{2} y^{2}+c^{\prime}{ }_{1} z+c^{\prime}{ }_{2} z^{2}\right) t \\
+\left(2 a a_{2}+2 b b_{2}+2 c c_{2}\right) t^{2}+\left(2 a a_{2}^{\prime}+2 b b_{2}^{\prime}{ }_{2}+2 c c^{\prime}{ }_{2}\right) t^{3}
\end{array}\right) t
$$

All $[K / L] t$-Padé approximants of (42) with $L \geq 1, K \geq 1$ and $K+L \leq 4$ give

$$
\left[\frac{K}{L}\right]_{\tilde{u}_{4}}(x, y, z, t)=\frac{\left(\begin{array}{r}
2 a_{1} b_{1} x y+2 a_{1} b_{2} x y^{2}+2 a_{1} c_{1} x z+2 a_{1} c_{2} x z^{2}+2 a_{2} b_{1} x^{2} y+2 a_{2} b_{2} x^{2} y^{2} \\
+2 a_{2} c_{1} x^{2} z+2 a_{2} c_{2} x^{2} z^{2}+2 b_{1} c_{1} y z+2 b_{1} c_{2} y z^{2}+2 b_{2} c_{1} y^{2} z+2 b_{2} c_{2} y^{2} z^{2}+a_{1}{ }^{2} x^{2} \\
+a_{2}{ }^{2} x^{4}+b_{1}{ }^{2} y^{2}+b_{2}{ }^{2} y^{4}+c_{1}{ }^{2} z^{2}+c_{2}{ }^{2} z^{4}+2 a_{1} a_{2} x^{3}+2 b_{1} b_{2} y^{3}+2 c_{1} c_{2} z^{3}
\end{array}\right) t}{a_{1} x+a_{2} x^{2}+b_{1} y+b_{2} y^{2}+c_{1} z+c_{2} z^{2}-\left(a_{1}^{\prime} x+a_{2}{ }_{2} x^{2}+b^{\prime}{ }_{1} y+b^{\prime}{ }_{2} y^{2}+c^{\prime}{ }_{1} z+c^{\prime}{ }_{2} z^{2}\right) t} .
$$

Now by changing $1 / s$ into $t$ in (3.12), we obtain $[K / L]_{\tilde{u}_{4}}$ in terms of $s$ as follow:

$$
\left[\frac{K}{L}\right]_{\tilde{u}_{4}}(x, y, z, t)=\frac{\left(\begin{array}{r}
2 a_{1} b_{1} x y+2 a_{1} b_{2} x y^{2}+2 a_{1} c_{1} x z+2 a_{1} c_{2} x z^{2}+2 a_{2} b_{1} x^{2} y+2 a_{2} b_{2} x^{2} y^{2} \\
+2 a_{2} c_{1} x^{2} z+2 a_{2} c_{2} x^{2} z^{2}+2 b_{1} c_{1} y z+2 b_{1} c_{2} y z^{2}+2 b_{2} c_{1} y^{2} z+2 b_{2} c_{2} y^{2} z^{2}+a_{1}{ }^{2} x^{2} \\
+a_{2}{ }^{2} x^{4}+b_{1}{ }^{2} y^{2}+b_{2}{ }^{2} y^{4}+c_{1}{ }^{2} z^{2}+c_{2}{ }^{2} z^{4}+2 a_{1} a_{2} x^{3}+2 b_{1} b_{2} y^{3}+2 c_{1} c_{2} z^{3}
\end{array}\right)}{\left(a_{1} x+a_{2} x^{2}+b_{1} y+b_{2} y^{2}+c_{1} z+c_{2} z^{2}\right) s-\left(a^{\prime}{ }_{1} x+a^{\prime}{ }_{2} x^{2}+b^{\prime}{ }_{1} y+b^{\prime}{ }_{2} y^{2}+c^{\prime}{ }_{1} z+c^{\prime}{ }_{2} z^{2}\right)} .
$$


Finally, when we apply the inverse Laplace transform to (30), it gives us an approximate solution. In fact, the approximate solution corresponds the exact solution.

$$
\tilde{u}_{4}(x, y, z, t)=u(x, y, z, t)=\left(a_{1} x+a_{2} x^{2}+b_{1} y+b_{2} y^{2}+c_{1} z+c_{2} z^{2}\right) e^{\left(\frac{a_{1}^{\prime} x+a^{\prime} 2 x^{2}+b_{1}^{\prime} y+b_{2}^{\prime} y_{2}^{2}+c_{1}^{\prime} z+c_{2} z^{2}}{a_{1} x+a_{2} x^{2}+b_{1} y+b_{2} y^{2}+c_{1} z+c_{2} z^{2}}\right) t} .
$$

\section{Conclusion}

In this study, LPRDTM, which is combining Laplace-Padé resummation method as a useful technique to find exact solutions and the RDTM, has been successfully applied to different types of $(1+3)$ dimensional wave equations. Our obtained results show that LPRDTM gives exact solutions of the equations by using only two iterations. Hence, LPRDTM is useful to ease CPU load and it helps us to have higher accuracy, efficiency and perfect harmony for solutions. Additionally, we point out that LPRDTM is very powerful and easy applicable mathematical tool for PDEs.

\section{Acknowledgement}

Thanks to Dr. Vural CAM and Ibrahim CALAN for their contributions in this study.

\section{Competing interests}

The authors declare that they have no competing interests.

\section{Authors' contributions}

All authors have contributed to all parts of the article. All authors read and approved the final manuscript.

\section{References}

[1] L. Debnath, Nonlinear Partial Differential Equations for Scientists and Engineers, Springer Science \& Business Media, 2011. https://books.google.com/books?id=Ir4yXgBesAsC\&pgis=1 (accessed December 10, 2014).

[2] A.M. Wazwaz, Partial Differential Equations and Solitary Waves Theory, Higher Education press and Springer Vergal, 2009.

[3] R.P. Agarwal, Difference Equations and Inequalities: Theory, Methods, and Applications, 2000. https://books.google.com/books?hl=tr\&lr=\&id=xMaAqOWMgXkC\&pgis=1 (accessed June 18, 2015).

[4] V.K. Srivastava, M.K. Awasthi, (1+n)-Dimensional Burgers' equation and its analytical solution: A comparative study of HPM, ADM and DTM, Ain Shams Eng. J. 5 (2014) 533-541.

[5] F. Mirzaee, M. Komak Yari, A novel computing three-dimensional differential transform method for solving fuzzy partial differential equations, Ain Shams Eng. J. (2015).

[6] M. Jafaryar, S.I. Pourmousavi, M. Hosseini, E. Mohammadian, Application of DTM for 2D viscous flow through expanding or contracting gaps with permeable walls, New Trends Math. Sci. 2 (2014) 145-158.

[7] M. Kurulay, M. Bayram, Approximate analytical solution for the fractional modified KdV by differential transform method, Commun. Nonlinear Sci. Numer. Simul. 15 (2010) 1777-1782.

[8] Y. Keskin, G. Oturanç, Reduced differential transform method for partial differential equations, Int. J. Nonlinear Sci. Numer. Simul. 10 (2009) 741-749.

[9] O. Acan, Y. Keskin, Approximate solution of Kuramoto-Sivashinsky equation using reduced differential transform method, in: Proc. Int. Conf. Numer. Anal. Appl. Math. 2014, AIP Publishing, 2015: p. 470003. doi:10.1063/1.4912680.

[10] O. Acan, Y. Keskin, Reduced differential transform method for (2+1) dimensional type of the Zakharov-Kuznetsov ZK(n,n) equations, in: Proc. Int. Conf. Numer. Anal. Appl. Math. 2014, AIP Publishing, 2015: p. 370015. doi:10.1063/1.4912604. 
[11] J. Yu, J. Jing, Y. Sun, S. Wu, On Finding Exact and Approximate Solutions to Some PDEs Using the Reduced Differential Transform Method, Appl. Math. Comput. 273 (2016) 697-705.

[12] A. Babaei, A. Mohammadpour, Solving an inverse heat conduction problem by reduced differential transform method Solving an inverse heat conduction problem by reduced differential transform method, New Trends Math. Sci. 3 (2016) 65-70.

[13] K. Yildirim, B. İbiş, M. Bayram, New solutions of the nonlinear Fisher type equations by the reduced differential transform, Nonlinear Sci. Lett. A. 3 (2012) 29-36.

[14] B. İbiş, M. Bayram, Approximate Solutions for Some Nonlinear Evolutions Equations By Using The Reduced Differential Transform Method, Intenational J. Appl. Math. Res. 1 (2012) 288-302.

[15] O. Acan, Y. Keskin, A Comparative Study of Numerical Methods for Solving (n+1) Dimensional and Third-Order Partial Differential Equations, J. Comput. Theor. Nanosci. (2016) (In press).

[16] Y. Zhang, Formulation and solution to time-fractional generalized Korteweg-de Vries equation via variational methods, Adv. Differ. Equations. 2014 (2014) 65.

[17] M.G. Sakar, F. Erdogan, A. Yldrm, Variational iteration method for the time-fractional Fornberg-Whitham equation, Comput. Math. with Appl. 63 (2012) 1382-1388.

[18] Q. Zhao, H. Xu, T. Fan, Analysis of three-dimensional boundary-layer nanofluid flow and heat transfer over a stretching surface by means of the homotopy analysis method, Bound. Value Probl. 2015 (2015) 64. doi:10.1186/s13661-015-0327-3.

[19] S. Sattari, M. Jahani, M. Gorji-bandpy, Application of different analytical methods to equation system of Bodewadt' s fixed disc and rotating stream, New Trends Math. Sci. 163 (2015) 149-163.

[20] M.G. Sakar, F. Erdogan, The homotopy analysis method for solving the time-fractional Fornberg-Whitham equation and comparison with Adomian's decomposition method, Appl. Math. Model. 37 (2013) 8876-8885.

[21] G. Akram, I.A. Aslam, Solution of fourth order three-point boundary value problem using ADM and RKM, J. Assoc. Arab Univ. Basic Appl. Sci. (2014). doi:10.1016/j.jaubas.2014.08.001.

[22] G.A. Baker Jr, Essentials of Padé approximants, Academic Press, UK, 1975.

[23] A.S.-R. Brahim Benhammouda, Hector Vazquez-Leal, Modified Reduced Differential Transform Method for Partial DifferentialAlgebraic Equations, 2014 (2014). doi:http://dx.doi.org/10.1155/2014/279481.

[24] S. Momani, G.H. Erjaee, M.H. Alnasr, The modified homotopy perturbation method for solving strongly nonlinear oscillators, Comput. Math. with Appl. 58 (2009) 2209-2220.

[25] P.-Y. Tsai, C.-K. Chen, An approximate analytic solution of the nonlinear Riccati differential equation, J. Franklin Inst. 347 (2010) $1850-1862$.

[26] A.E. Ebaid, A reliable aftertreatment for improving the differential transformation method and its application to nonlinear oscillators with fractional nonlinearities, Commun. Nonlinear Sci. Numer. Simul. 16 (2011) 528-536. 